$02-880$

ORNL-4984

\title{
CONVERGENCE OF THE DISCRETE ORDINATES METHOD FOR THE TRANSPORT EQUATION
}

\author{
P. M. Anselone
}

A. G. Gibbs 


\section{Printed in the United States of America. Avallabie from National Tecnnical Information Service U.S. Liepartment of Commerce \\ 5285 Port Royal Fioad. Springfield, Virginia 22151}

Price Printed Copy \$4.00. Microfiche $\$ 1.45$

This report was prepared as an account of Nork sponsored by the United S:ates Governitenit. iveitiver the United States nor the United States Atomic Energy Commission, nor ariy of theip employees, nor any of their contractors, subcontractors, or their einployees, makes any warranty, express or implied, or assumes any legal liability or responsitulity for the accuracy. completeness or usefuiress of any informatior. apparatus, p:oduct or process disclosed, or represents that it' use would not infringe privately owned rights. 


\section{ORNL -4984 \\ UC-32 - Mathematics and isaputers}

Contract No. W-7405-eng-26

COMPUTER SCIENCES DIVISIGN

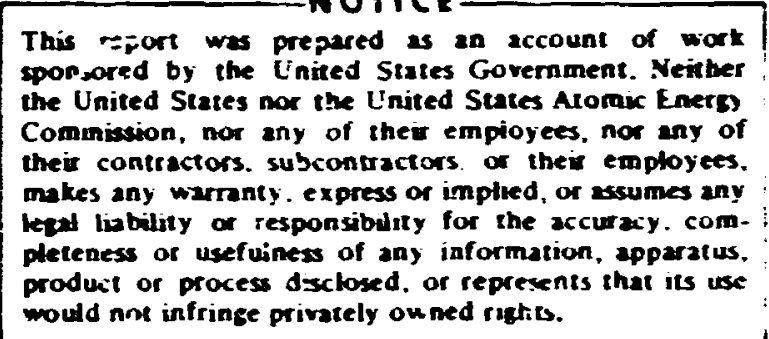

CONJERGEHCE OF THE DISCREFE ORDTHATES

MEIHOD FOR THE TRANSPORT EQUATION

P. M. Arzelone ${ }^{2}$ and A. G. Gibbs ${ }^{3}$

\section{JULY 1974}

${ }^{1}$ ipported by the U. S. Atomic Energy Commission at the Pacific Northwest Laboratory, Richland, Washington, and the Union Carbide Corporation, Miclear Division, Oak Ridge, Tennessee ${ }^{2}$ Consultant. Oregon State University, Corvaliis, Oregon ${ }^{3}$ Battelle, Pacific Northwest Laboratory, Richland, Washingtcn

OAK RIDGE NATIONAL IABCRATORY

Oak Ridge, Tennessee 37830

$$
\text { operated by }
$$

UNION CARBIDE CORPIRATION

for the

U. S. ATOMIC EHERGY CCMMISSION 
Convergence of the Discrete Ordinates

Method for the Transport Equation

P. M. Anselone and A. G. Gibbs

ABSTRACT

The transport equation for the distribution of neutrons or radiation is an integro-differential equation of some complexdty. The discrete ordinates method of approximate solution, which is based on numerical integration, has been used for a number of years to obtain satisfactory results. However, a complete convergence of error analysis is available only in some rather idealized cases. We survey the rarious contributions to the literature on the convergence question from an abstract and unifying point of view. In the process, methods of analysis are developed which are applicable to very general transport problems.

1. Introduction

The transport equation (transfer equation, linear Boltzmann equation) governs the distribution of neutrons in a nuclear reactor or of radiation in a stellar or planetary atmosphere. It is an integro-differential equation of some compiexity in its more general forms. Primary independent variables are position and direction, speed (alternatively energy, frequency or wavelength). plus possibly time. The differential operator in the equation describes attenuation due to both absorption and scattering away from a particular direction. The integral operator describes scattering into a particular direction from other directions and production by fission. Source terms may be present. On any boundary surface there are boundary conditions corresponding to incoming directions. For time-dependent problems there are initial conditions. 
BLANK PAGE 
Since the transport equation can be solved explicitly only in a few very special cases, approximation methods are commonly used. The discrete ordinates method involves the replacement of the integral operator by a numerical integral operator in order to obtain differential equations for approximate solutions. Empirically, this method yields very satisfactory results for a large class of transport problems. However, a complete and rigorous convergence and error analysis is prcsently available only ir some ratiner idealized cases.

The discrete ordinates method was first applied by Wick [18] and by Chandrasekhar [7, 8] to particular steady-staic problems from astrophysics with homogeneous media bounded by single planes or parallel planes (halfspaces or slabs), usually urder conditions of isotropic scattering and isotropic sources. Convergence theorems for the approximations were obtained for various problems of this type with isotropic scattering by Anselone [1-5], Kofink [13], Keller [10, 11] and Wendroff [17]. Subsequently. convergence theorems have been obtained for certain transport problems involving anisotropic scattering, other geometries, and time-depencence, by Keller [12], Nestell [16], Madsen [14] and wilson [20].

We shall survey these contributions from an abstract and unifying point of view in order to isolate what is essential to the convergence of the discrete ordinates approximations. In the process, methods of analysis 
will be developed which appear to be applicable to very general transport problems and to other integrodifferential equations. The discussion is in terms of a sequence of model. problems of increasing complexity. All functions considered will be real. For mathematical convenience and physical relevence specified functions are continuous and usually nonnegative. We remark that the problems surveyed here involve discrete ordinates approximations only for the angular integrals, and thus do not represent the most general case of discretization as practiced, say, in many nuclear reactor applications today, where the spatial variable is also discretized. However, as noted below, the powerful analytical tools developed in applications to angular discrete ordinates approximations also show promise for investigations of the more general case.

2a. An Isotropic Transport Problem in a Finite Slab Corsider the problem for $\phi(x, \mu)$ with $a \leq x \leq b$, $-1 \leq i \leq 1$, given by (2.1)

$$
-\frac{\partial \phi\left(x, \psi \psi^{\prime}\right.}{\partial x}+\phi(x, \mu)=\frac{c}{2} \int_{-1}^{1} \varphi\left(x, \psi^{\prime}\right) d \mu^{\prime}+g(x) .
$$

$$
\hat{i}(a, i)=0 \text { for } i>c, \phi(b, i)=0 \text { for } \mu<0,
$$

where $\phi(x, \mu)$ is the one-speed angular flux at depth $x$ in a homogeneous slab in a direction making an ar.gle $\theta=\cos ^{-1} \mu$ with the positive $x$-axis. The positive 
number $c$ is of order $I$ and represents the mean number of secondary neutrons per collision. In the above equation, distance has been measured in units of neutron mean free paths, i.e., $x=\sigma \tau$, where $\sigma$ is the total cross section and $\tau$ is the actual distance.

The boundary conditions correspond to a situation with no reflection of neutrons at the surface and no incident neutrons. Actually, a non-zero incident flux boundary condition could have been assumed. However, such a problem can be reduced to (2.1) for $\phi_{1}=\phi-\phi_{0}$. where $\phi_{0}$ satisfies the homogeneous equation

$$
u \frac{\partial \Phi_{0}(x, \mu)}{\partial x}+\phi_{0}(x, u)=0
$$

and the given boundary conditions. If this reduction is not made, then an unnecessary approximation is introduced for $\phi_{0}$, which can be found explicitly. For the same reason, zero boundary conditions can and should be assumed also in applications of the discrete ordinates method to more general transport problems.

To proceed, assume a numerical integration rule such that, for any continuous function $h(i),-1 \leq \mu \leq 1$,

$$
\begin{aligned}
& \sum_{j= \pm 1}^{ \pm n} w_{n j} h\left(u_{n j}\right) \rightarrow \int_{-1}^{i} h(i) d i, n \rightarrow \infty, \\
& w_{n,-j}=w_{n, j}>0, \mu_{n,-j}=-j_{n, j} \cdot \\
& j<i_{n 1}<i_{n 2} \cdots<j_{n n} \leq 1 .
\end{aligned}
$$


The Gauss quadrature formula is an example. The symmetry conditions on the $w_{n j}$ and $i_{n j}$ are a convenience rather than a necessity.

Discrete ordinates approximations ${ }_{\psi_{n}}(x, \mu)$, $\mathrm{n}=1,2, \ldots$, satisfy

$$
\mu \frac{\partial \phi_{n}(x, \mu)}{\partial x}+\phi_{n}(x, \mu)=\frac{c}{2} \sum_{j= \pm 1}^{ \pm n} w_{n j} \phi_{n}\left(x, \mu_{n j}\right)+g(x),
$$

$$
\phi_{\mathrm{n}}(\mathrm{a}, \mu)=0 \text { for } \mu>0, \leftarrow_{\mathrm{n}}(\mathrm{b}, \mu)=0 \text { for } \mu<0 \text {. }
$$

For $\mu=\mu_{n i}, i= \pm 1, \ldots, \pm n$, this is a system of ordinary differential equations for $\dot{\hat{\varphi}}_{n}\left(x, \mu_{n i}\right)$. Then (2.2) yields $\phi_{n}(x, \mu)$.

The problems for $\phi$ and $\phi_{\mathrm{n}}$ have equivalent integral equation formulations which are more convenient for the convergence analysis. Let

$$
\begin{aligned}
& f(x)=\frac{c}{2} \int_{-1}^{1} \phi(x, j) d \mu+g(x), \\
& f_{n}(x)=\frac{c}{2} \sum_{j= \pm 1}^{ \pm n} w_{n j} \phi\left(x, i_{n j}\right)+g(x) .
\end{aligned}
$$

From (2.1) and (2.3), it foilows that

$$
\phi(x, 0)=f(x),
$$

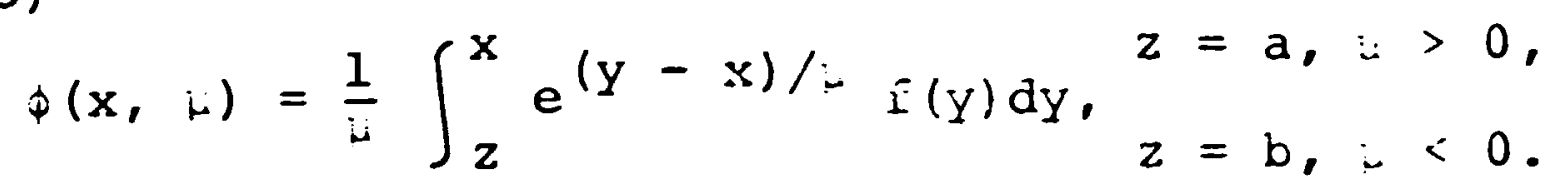

The equations for $\Phi_{n}$ in terms of $f_{n}$ are identical. From (2.3) and (2.5), 
(2.6) $f(x)-\frac{c}{2} \int_{a}^{b} E_{1}(|x-y|) f(y) d y=g(x)$,

where $E_{1}$ is the exponential integral function of order sne,

(2.7) $\quad E_{1}(s)=\int_{0}^{1} e^{-s / \mu} \mu^{-1} d \mu, s>0$,

Which has a logarithmic singularity at $s=0$. Similarly,

(2.8) $f_{n}(x)-\frac{c}{2} \int_{a}^{b} E_{n l}(|x-y|) f_{n}(y) d y=s(x)$,

where $E_{n l}$ is a numerical integration approximation to $E_{1}$ :

(2.9) $\quad E_{n l}(s)=\sum_{j=1}^{n} w_{n j} \frac{e^{-s / \mu_{n j}}}{\mu_{n j}}, s \geq 0$,

(2.10) $E_{n 1}(s) \rightarrow E_{1}(s)$ uniformly for $s \geq \varepsilon$ as $n \rightarrow \infty$

for each $\varepsilon>C$. Thus, the discrete ordinates method is seen to be equivalent to the approximation of the integral operator in (2.6) by the integral operator in (2.8).

Express (2.6) and (2.8) in operator form

$$
(I-K) f=g, \quad\left(I-K_{n}\right) f_{n}=g
$$

on the space $C[a, b]$ with the max norm. Then $K$ and $\mathrm{K}_{\mathrm{n}}$ are Fredholm integral operators with kernels

$$
k(x, y)=\frac{c}{2} E_{1}(|x-y|), \quad k_{n}(x, y)=\frac{c}{2} E_{n 1}(|x-y|) .
$$


These are bourded Iinear operators on $C[a, b]$ and for c not too large (the case of a subcritical medium, assumed here) we: have

$$
\|k\|=\max _{x} \int_{a}^{b} k(x, y) d y<1
$$

Hence, $(I-K)^{-1}$ exists,

$$
\left\|(I-K)^{-1}\right\| \leq(1-\|K\|)^{-1}
$$

and $f$ and $\phi$ are uniquely determined.

From (2.10),

$\left\|k_{n}-k\right\| \rightarrow 0, \quad\left\|k_{n}\right\| \rightarrow \| k_{\|}$as $n \rightarrow \infty$.

Thus, we are in the realm of standard operator approximation theory. For $n$ sufficiently large, $\left\|k_{n}\right\|<1$, $\left(I-X_{n}\right)^{-1}$ exists and is bounded uniformly in $n$, and (2.12) $\left(I-K_{n^{\prime}}{ }^{-1}-(I-K)^{-1}=\left(I-K_{n}\right)^{-1}\left(K_{n}-K\right)(I-K)^{-1}\right.$, (2.13) $\left\|\left(I-K_{n}\right)^{-1}-(I-K)^{-1}\right\| \leq\left\|\left(I-K_{n}\right)^{-1}\right\|\left\|K_{n}-K\right\|\left\|(I-K)^{-1}\right\|$,

(2.14) $\quad\left\|\left(I-K_{n}\right)^{-1}-(I-K)^{-1}\right\| \leq \frac{\left\|(I-K)^{-1}\right\|^{2}\left\|K_{n}-K\right\|}{1-\left\|(I-K)^{-I}\right\|\left\|K_{n}-K\right\|}$,

(2.15) $\left\|\left(I-K_{n}\right)^{-1}-(I-K)^{-1}\right\| \leq \frac{\left\|\left(I-K_{n}\right)^{-1}\right\|^{2}\left\|K_{n}-K\right\|}{1-\left\|\left(I-K_{n}\right)^{-1}\right\|\left\|K_{n}-K\right\|}$, (2.16) $\quad\left\|\left(I-K_{n}\right)^{-1}-(I-K)^{-1}\right\| \rightarrow 0$ as $n \rightarrow \infty$.

The error bound in (2.14) is "theoretical" in the sense 
that it involves $(I-K)^{-1}$, whereas $(2.15)$ is "Fractical" because it deperds on $\left(I-K_{n}\right)^{-1}$, and can thus be computed. The bound (2.13) is of mixed type. It follows from (2.16) and (2.5) fif that $f_{n} \rightarrow f$ and $\phi_{n} \rightarrow \phi$ uniformly as $\mathrm{n} \rightarrow \infty$.

This is essentially the path followed by Anselone $[4,5]$ and by Keller [11], although Keller's analysis was less abstract and he defined the discrete ordinates approximations only at the quadrature points. Previously, Keller [10] and Wendroff [17] treated the differential operators directly without inverting them. The results were less satisfactory: $\mathrm{L}^{2}$ convergence on quadrature points, and uniform convergence on quadrature points under a restrictive assumption. Kofink [13] established $\mathrm{L}^{2}$ convergence on quadrature points by exploiting an equivalence between the discrete ordinates method and the spherical harmonics method.

2b. An Isotropic Transport Problem in a Half Space Anselone [1-4] also derived uniform convercience theorems for isotropic transport problems in the case of semi-infinite slabs $(0 \leq x \leq \infty)$. In particular, the classical Milne problem leads to the homogeneous equation $(I-K) f=0$, where $K$ is the same integral operator as above. The Wiener-Hopf methcd, based on Fourier transforms, was devised originally to solve this equation. 
A more direct method, which anticipated later theories of positive and monotone operators, was given by Hopf [9] for the ase $c=?$ It involves a change of variable $f(x)=x+g(x)$. Then $q$ satisfies the inhomogeneous equation $(I-K) q=E_{3}$, where $E_{3}$ is the exponential integral function of order 3 ,

$$
E_{3}(s)=\int_{0}^{I} e^{-s / \mu} \mu d_{j}, s \geq 0
$$

A nonnegative solution $\mathrm{q}$ is sought in the space of bounded continuous functions on $(0, \infty)$. Although now $\|\mathrm{k}\|=1$, monotonicity considerations yield a unique solution $q$ given by the uniformly convergent Neumann series

$$
q=\sum_{m=0}^{\infty} K^{m} E_{3}
$$

The discrete ordinates approximation problem can be recast in the form $\left(I-K_{n}\right) f_{n}=0$. Let $f_{n}(x)=x+g_{n}(x)$. Then $\left(I-k_{n}\right) g_{n}=E_{n 3}$, where $E_{n 3}$ is a numerical integration approximation to $E_{3}$, and $q_{n}$ is given by

$$
q_{n}=\sum_{m=0}^{\infty} k_{n}^{m} E_{n 3} .
$$

A detailed term by term analysis yields $q_{\mathrm{n}} \rightarrow q$ uniformly as $\mathrm{n} \rightarrow \infty$. It follows that $\mathrm{f}_{\mathrm{n}} \rightarrow \mathrm{f}$ and $\dot{\phi}_{\mathrm{n}} \rightarrow \phi$ iniformly.

3a. An Anisotropic Transport Problem in a Finite Slab Consider the problem for $\phi(x, \mu)$ with $a \leq x \leq b$, $-1 \leq \mu \leq 1$, given by 
$(3.1)$

$$
\mu \frac{\partial \phi(x, \mu)}{\partial x}+\phi(x, \mu)=\frac{1}{2} \int_{-1}^{1} p\left(x, \mu, \mu^{\prime}\right) \phi\left(x, \mu^{\prime}\right) d \mu^{\prime}+g(x, \mu),
$$$$
\phi(a, j)=0 \text { for } \mu>0, \phi(b, \mu)=0 \text { for } \mu<0 \text {. }
$$

Here, $x$ represents the optical depth measured from the plane of the origin, i.e.,

$$
x(\tau)=\int_{0}^{i} \sigma\left(\tau^{\prime}\right) d \tau^{\prime}
$$

with $\sigma(\tau)$ the total cross section at position $\tau$, and $\tau$ the distance measured from 0 . The cifferential kernel $p\left(x, \mu, \nu^{\prime}\right) d \mu$ represents the average number of neutrons emerging with direction cosines in di, following a collision by a neutron with direction cosine $;:^{\prime}$ at position $x$. The function $g$ represents sources within tine slab. We assume here that bcth $p$ and $g$ are continuous functions of $x$ and $\mu$.

Let

(3.2) $f(x, \mu)=\frac{1}{2} \int_{-1}^{1} p\left(x, \mu, \mu^{\prime}\right) \phi\left(x, \mu^{\prime}\right) d \mu^{\prime}$.

Then the problem is expressed in operator form as

$$
D \xi=f, \quad f=L \Phi+g,
$$

where $f, g \in C(x)$ with $x=[a, b] \times[-1,1]$. Under reasonable conditions on $\phi, M=D^{-1}$ exists as an operator on $C(X)$, and $\phi=M f=D^{-1} f$ is given by 


$$
\phi(x ; 0)=f(x, 0)
$$

(3.4)

$$
\varphi(x, \mu)=\frac{1}{\mu} \int_{z}^{x} e^{\left(x^{\prime}-x\right) / i} f\left(x^{\prime}, j\right) d x^{\prime}, \quad z=a \text { for } \mu>0,
$$

Therefore, an equivalent formulation of the problem is

$$
Q=M f, \quad(I-K) f=g, \quad K=L M .
$$

It can be shown that $\mathrm{K}$ is a bounded integral operator which maps $C(X)$ into $C(X)$. Since $K$ has a nonnegative kernel

$$
\|k\|=\|\operatorname{ke}\|, \quad \text { e } \in C(x), \quad e \equiv 1
$$

If $\mathrm{p}$ is not too large, then $\|\mathrm{k}\|<1$, which we assume in what follows. Then $(I-K)^{-1}$ exists as a bo:unded operator on $C(X)$, and $f, \hat{\psi}$ are uniquely determined.

Assume a convergent, positive quadrature rule:

$$
\begin{aligned}
& \sum_{j=1}^{n} w_{n j} h\left(\mu_{n j}\right) \rightarrow \int_{0}^{1} h(j) d_{j i} \text { as } n \rightarrow \infty, \quad h \in C[-1,1], \\
& w_{n j}>0, \quad 1 \leq j \leq n, \quad n=1,2, \ldots .
\end{aligned}
$$

In particular, for $h=e \equiv 1$,

$$
\sum_{j=1}^{n} w_{n j} \rightarrow 1 \text { as } n \rightarrow \infty
$$

Hence, there exists $B<\infty$ such that

$$
\sum_{j=1}^{n} w_{n j} \leq B, \quad n=1,2, \ldots .
$$


The discrete ordinates approximations $\phi_{n}$ satisfy

$$
\mu \frac{\partial \phi_{n}(x, \mu)}{\partial x}+\phi_{n}(x, \mu)=\frac{1}{2} \sum_{j=1}^{n} w_{n j} p\left(x, \mu, \mu_{n j}\right) \phi\left(x, \mu_{n j}\right)+g(x, \mu),
$$

$$
\phi_{n}(a, \mu)=0 \text { for } \mu>0, \quad \phi_{n}(b, \mu)=0 \text { for } \mu<0 \text {. }
$$

Equivalent formulations are

$$
\begin{array}{ll}
D \phi_{n}=f_{n^{\prime}} & f_{n}=L_{n} \phi_{n}+g, \\
\phi_{n}=M f_{n^{\prime}} & \left(I-K_{n}\right) f_{n}=g, \quad K_{n}=L_{n} M
\end{array}
$$

where $K_{n}$ is a bounded linear operator on $c(x)$. since the "kernel" of $k_{n}$ is positive, $\left\|k_{n}\right\|=\left\|k_{n} e\right\|$. where $e \equiv 1$.

It follows from (3.5) inat $k_{n} \rightarrow k, i . e .$, $\left\|k_{n} h-k h\right\|+0$ as $n \rightarrow \infty$ for each $h e c(x)$. However, $\left\|k_{n}-k\right\|+0$ in the anisotropic case, so the standard operator approximation theory used in section 2 above is not applicable. An alternative course of action is pursued here.

It follows from $k_{n} \rightarrow K,\|k\|=\|k e\|$ and $\left\|k_{n}\right\|=\left\|K_{n} e\right\|$ that $\left\|\mathrm{k}_{\mathrm{n}}\right\| \rightarrow\|\mathrm{k}\|$. Recall. that $\|\mathrm{k}\|<1$. Hence, for $n$ sufficiently large, $\left\|k_{n}\right\|<1,\left(I-k_{n}\right)^{-1}$ exists and is bounded uniformly in $n$, and

$$
\begin{aligned}
& \left(I-K_{n}\right)^{-1}-(I-K)^{-I}=\left(I-K_{n}\right)^{-1}\left(K_{n}-K\right)(I-K)^{-1}, \\
& f_{n}-f=\left(I-K_{n}\right)^{-1}\left(K_{n} f-K f\right),
\end{aligned}
$$


(3.11) $\quad\left\|E_{n}-f !:\right\|\left(1-k_{n}\right)^{-1}\|\| k_{n} f-k f \|$.

It follows that $f_{n} \rightarrow f$ and $i_{n} \rightarrow+$ uniformly as $n \rightarrow \infty$. the error bound in (3.11) is of mixed type, neither purely theoretical ror purely practical.

The fact that $k$ and $k_{n_{l}}$ are positive operators is essential to the foregoing analysis, which is an abstract version of that carried out by Keller [12] in a more classical spirit.

3b. An Anisotropic Transport Problem in a Half Space

We remark that the case of anisotropic scattering problems ir. a half-space was treated by Nestell [16] by an adaptation of the positive operator theory of Hopf. That work is an extension of the isotropic scattering problem discissed in section $2 b$, above.

4. Collectively Compact Operator Approximation Theory We shall consider the same problem as in 3 from another point of view, which yields both theoretical and practical (computable) error bounds. The analysis is based on collectively compact operator approximation theory (cf. Anselone [6]).

It can be shown that the operator $K$ in (3.4) is compact: $\{\mathrm{Kh}:\|\mathrm{h}\| \leq 1\}$ is relatively compact or, equivalently, bounded and equicontinuous. The secuence $\left\{k_{n}: n \geq 1\right\}$ is collectively compact: $\left\{k_{n} h:\|h\| \leq 1, n \geq 1\right\}$ is relatively compact. Thus, we have 
$\mathrm{K}_{\mathrm{n}} \rightarrow \mathrm{K}, \mathrm{K}$ compact, $\mathrm{K}_{\mathrm{n}}$ collectively compact.

These are the hypotheses for a general operator approximation theory in a Banach space setting. Some of the main conclusions of the theory are as follows.

The operator $(I-K)^{-1}$ exists iff for $n$ sufficiently large $\left(I-K_{n}\right)^{-1}$ exists and is bounded uniformly in $n$, in which case

$$
\left(I-K_{n}\right)^{-1} \rightarrow(I-K)^{-1} \text {. }
$$

Let $f=(I-K)^{-1} g$ and $f_{n}=\left(I-K_{n}\right)^{-1} g$. Then $\left\|f_{n}-f\right\| \rightarrow 0$. Let

$$
\begin{aligned}
& \Delta_{n}=\left\|(I-K)^{-1}\right\|\left\|\left(K_{n}-K\right) K_{n}\right\|, \\
& \Delta^{n}=\left\|\left(I-K_{n}\right)^{-1}\right\|\left\|\left(K_{n}-K\right) K\right\| .
\end{aligned}
$$

Then

$$
\begin{gathered}
\Delta_{n} \rightarrow 0, \Delta^{n} \rightarrow 0 \text { as } n \rightarrow \infty, \\
\left\|f_{n}-f\right\| \leq \frac{\left\|(I-K)^{-1}\right\|\left\|k_{n} g-K g\right\|+\Delta_{n}\|f\|}{1-\Delta_{n}} \text { for } \Delta_{n}<1, \\
\left\|f_{n}-f\right\| \leq \frac{\left\|\left(I-K_{n}\right)^{-1}\right\|\left\|K_{n} g-K g\right\|+\Delta^{n}\left\|f_{n}\right\|}{1-\Delta^{n}} \text { for } \Delta^{n}<1 .
\end{gathered}
$$

Moreover, $(I-K)^{-1}$ exists whenevex $\left(I-K_{n}\right)^{-1}$ exists and $\Delta^{\mathfrak{n}}<1$. Thus the existence of $f=(I-K)^{-1} g$ can be inferred from approximations. 
The convergence of the discrete ordinates approximations was established by means of the collectively compact theory by Nestell [16] for an anisotropic transport problem in a finil. slab with $p=p\left(\mu, \mu^{\prime}\right)$ and by Nelson [15] in greater generality. Both authors worked directly with explicit representations of $K$ and $k_{n}$. Siñce $\bar{k}$ is an integral operator on functions of two variables, its kernel involves four variables. The kernel has a weak singularity. The definition of $k_{n}$ is similar, with the $\mu$-integral replaced by a sum. The complications of $k$ and $k_{n}$, particularly the singularity, make it difficult to see what makes the analysis go through. We propose a different approach here, which is more transparent and extends more readily to a larger class of transport problems. By (3.5) and (3.7),

$$
K=L M, \quad K_{n}=L_{n} M
$$

where $M$ is defined in (3.4) and

$$
\begin{aligned}
& (L \phi)(x, \mu)=\frac{1}{2} \int_{-1}^{1} p\left(x, \mu, \mu^{\prime}\right) \phi\left(x, \mu^{\prime}\right) d \mu^{\prime}, \\
& \left(L_{n} \phi\right)(x, \mu)=\frac{1}{2} \sum_{j=1}^{n} w_{n j} p\left(x, \mu, \mu_{n j}\right) \phi\left(x, \mu_{n j}\right) .
\end{aligned}
$$

The basis of the analysis is to take advantage of the fact that only L, which has a continuous kernel, is approximated in order to define $K_{n}=L_{n} M$. The singularity comes from $M$, which can be treated separately. 
Consider $\phi=$ Mf for $f$ e $C(x ;$. From (3.4),

$$
\begin{aligned}
& \phi(x, 0)=E(x, 0), \\
& \phi(x, \mu)=\left\{\begin{array}{l}
\frac{(x-z)}{\mu} e^{-t} f(x-t \mu, \mu) d t, \quad z=a \text { for } \mu>0, \\
z=b \text { for } \mu<0 .
\end{array}\right.
\end{aligned}
$$

It follows that $\phi$ is continuous except perhaps at $(a, 0)$ and $(b, 0)$. The boundary conditions

$$
\phi(a, \mu)=0 \text { for } \mu>0, \phi(b, \mu)=0 \text { for } \mu<0 \text {, }
$$

are satisfied and

$$
\begin{array}{ll}
\phi(x, \mu) \rightarrow \phi(a, 0) & \text { as } x \rightarrow a, \mu \rightarrow 0-, \\
\phi(x, \mu) \rightarrow \phi(b, 0) & \text { as } x \rightarrow b, \mu \rightarrow 0+, \\
\phi(x, \mu)-\left(1-e^{(a-x) / \mu}\right) \phi(a, 0) \rightarrow 0 & \text { as } x \rightarrow a, \mu \rightarrow 0+, \\
\phi(x, \mu)-\left(1-e^{(b-x) / \mu}\right) \phi(b, 0) \rightarrow 0 & \text { as } x \rightarrow b, \mu \rightarrow 0-.
\end{array}
$$

Thus, $R(M)$, the range of $M$, consists of bounded functions which are continuous except perhaps at two points. By routine arguments, $L$ and $L_{n}$ map $R(M)$ into $C(X)$. Define the domains of $L$ and $L_{n}$ by $D(L)=D\left(L_{n}\right)=R(M)$. Then the operators $K=L M$ and $K_{n}=L_{n} M \operatorname{map} C(X)$ into $C(X)$. It follows easily from $K_{n}-K=\left(L_{n}-I\right) M$ and the properties of $R(M)$ that $K_{n} \rightarrow K$. To prove tinat $K$ is compact and $\left\{k_{n}\right\}$ is collectively compact, consider $K f=L M f$ and $K_{n} f=L_{n}$ iff for $\|f\| \leq 1$. As above, let $\phi=$ Mf. Then for any $\varepsilon>0$ and any $\nu>0$ there 
exists $\delta=\delta(\varepsilon, v)$ independent of $f$ such that $\left|\phi(x, \mu)-\phi\left(x^{\prime}, \mu\right)\right|<\varepsilon$ for $\left|x-x^{\prime}\right|<\delta,|\mu|>\nu$. This is an equicontinuity property in $x$, uniform for $|\mu|>v$. Essencially, $M$ is a compact operator with respect to $x$, uniformiy for each $v>0$. Similarly, the operator $\mathrm{L}$ is compact with respect to $\mu$ uniformly for $a \leq x \leq b$. Examination of $(L \phi)(x, \mu)-(L \phi)\left(x, \mu^{\prime}\right)$ and $(L \phi)(x, \mu)-(L \phi)\left(x^{\prime}, \mu\right)$ reveals that the sets $\{$ Kf $=$ LMf $:\|f\| \leqq 1\}, \quad\left\{K_{n} f=L_{n} M f:\|f\| \leqq I, n \geq 1\right\}$ are bounded and equicontinuous. Therefore, $K$ is compact and $k_{n}$ is collectively compact.

The error bounds in the collectively compact theory depend particularly on

$$
\begin{aligned}
& \left\|\left(K_{n}-K\right) g\right\|=\left\|\left(L_{n}-L\right) M g\right\|, \\
& \left\|\left(K_{n}-K\right) K\right\|=\left\|\left(I_{n}-L\right) M L M\right\|, \\
& \left\|\left(K_{n}-K\right) K_{n}\right\|=\left\|\left(L_{n}-L\right) M L_{n} M\right\| .
\end{aligned}
$$

Calculations based on tisese equations should yield sharper bounds than would be obtained otherwise.

5. A Three-Dimensional Anisotropic Transport Problem Let $\Gamma$ be a domain in $R^{3}$ with bourdary $\partial \Gamma$ which, at least piece-wise, has a well-defined tangent plane at each point. Directions will be denoted by $\Omega \in \delta$, the 
anit sphere in $R^{3}$. Consider the problem for $\phi(x, \Omega)$, $x \in \Gamma, \Omega \in S:$

$$
\Omega \cdot \nabla \phi(\mathbf{x}, \Omega)+\sigma(\mathbf{x}, \Omega) \phi(\mathbf{x}, \Omega)=\mathbf{E}(\mathbf{x}, \Omega) .
$$

$$
f(x, \Omega)=\int_{\delta} p\left(x, \Omega, \Omega^{\prime}\right) \phi\left(x, \Omega^{\prime}\right) d \Omega^{\prime}+g(x, \Omega),
$$

with $\phi(x, \Omega)=0$ for $x \in \partial \Gamma$ and $\Omega$ an incoming direction. The gradient operator $\nabla$ acts with respect to $x$. Assume that $f, g$ and $p$ are continuous and and that $\mathrm{p}$ is nonnegative. In operator form (5.1) becomes

$$
D \phi=E, \quad f=L \phi+g \text {. }
$$

Motivated by the fact that $\Omega \cdot \nabla$ is the directional derivative in the direction $\Omega$, we introduce additional notation as illustrated in the following diagram.

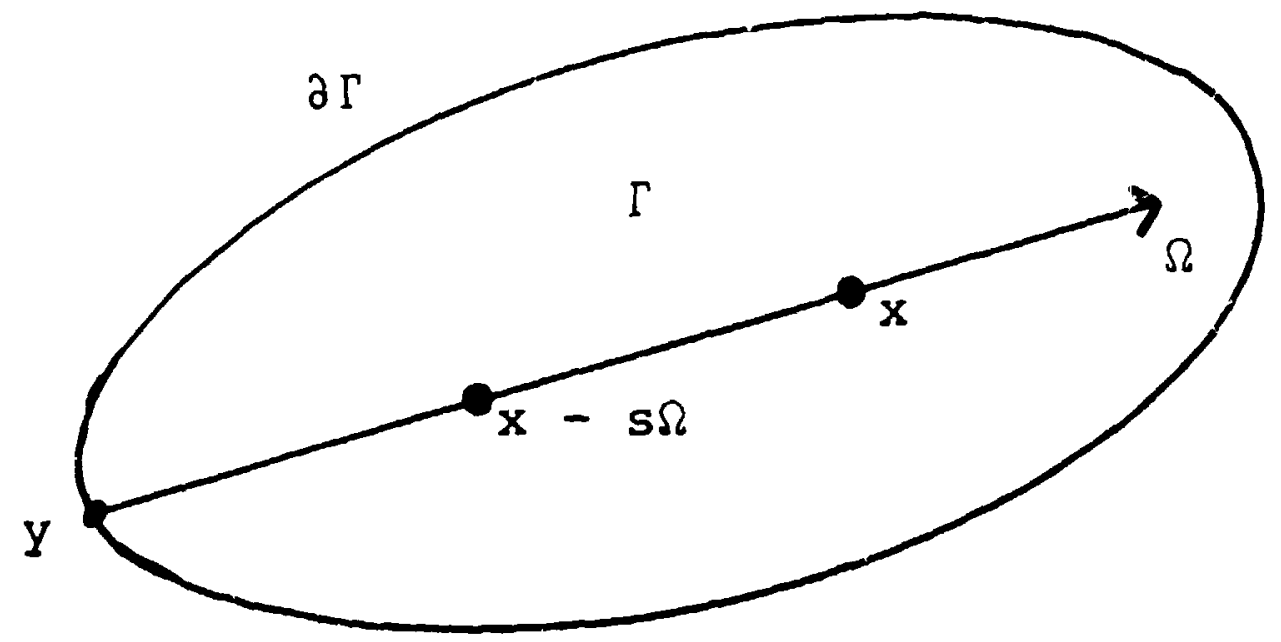

The ray through $\mathrm{x}$ in the direction $-\Omega$ is given by $x-s \Omega, s \geq 0$. Let $d(x, \Omega)=\|x-y\|_{2}$, the Euclidean distance from $x$ to $\partial \Gamma$ along the $r a y$. 
An equivaient formulation of $D \phi=f$ is

$(5.3)-\frac{d}{d s} \phi(x-s \Omega, \Omega)+\sigma(x-s \Omega, \Omega) \phi(x-s \Omega, \Omega)=f(x-s \Omega, \Omega)$.

Solve (5.3) to obtain

(5.4) $\phi(x, \Omega)=\int_{0}^{d(x, \Omega)} f(x-s \Omega, \Omega) \in \int_{0}^{-\int_{0}^{s} \sigma(x-r \Omega, \Omega) d r} d s$.

Thus, $M=D^{-1}$ exists as an operator on $C(X)$, where $X=\vec{\Gamma} \times \delta$, and $\phi=M f$ is given by (5.4). The originaI problem now becomes
$(5.5)$
$\phi=\mathrm{Mf}$,
$(I-K) f=g$,
$\mathrm{K}=\mathrm{LM}$,

where $L$ is the integral operator in (4.1).

Discrete ordinates approximations $\phi_{n}(x, \Omega)$ satisfy

$$
D \phi_{n}=f_{n}, \quad f_{n}=L_{n} \phi_{n}+g,
$$

where $I_{n}$ is a numerical integration approximation to $L$ defined in terms of a positive convergent quadrature rule. Now-familiar reasoning yields the equivalent formulation of (5.6),

$$
\phi_{n}=M f_{n^{\prime}} \quad\left(I-K_{n}\right) f_{n}=g, \quad K_{n}=L_{n} M .
$$

The tasks are the same as before, namely to prove that $(I-K)^{-1}$ exists, $\left(I-K_{n}\right)^{-1}$ exists for $n$ sufficientiy large, and

$$
\left(I-K_{n}\right)^{-i} \rightarrow(I-K)^{-1},
$$


which implies that $f_{n}-I$ and $\phi_{n} \rightarrow \phi$ uniformly. If $\|k\|<1$ and $k_{n} \rightarrow K$, then these conclusions follow as in section 3. If also $k$ is compact and $\left\{k_{n}\right\}$ is collectively compact, then the error bounds of Section 4 are obtained. This program will be undertaken in a forthcoming sequel.

The collectively compact operator approximation theory may also be applicable to the case where the approximations are made with respect to the spatial variable, either by approximating the detailed variatior. of $\sigma(x, \Omega)$ and $p\left(x, \Omega, \Omega^{\prime}\right)$ with respect to position $x$ by appropriate smoothed functions, and/or by carrying out the spatial integrations by some quadrature approximation. In this more general setting, we have $M$ approximated by $M_{j}$ as well as $L$ approximated by $L_{n}$. Thus, it will be recessary to consider $L_{n}{ }_{j} \rightarrow L M$ in an appropriate sense.

6. A Positive Operator Approach

Explicit results for the three dimensional problem can $b \in$ obtained rather easily if an additional assumption is made. Consider the problem of section 5 in the form
(6.1)
$\mathrm{T} \phi=g$,
$T=D-L$

under the restriction

(6.2) $\sigma\left(x, s^{\prime}\right)-\int_{\mathrm{g}} \mathrm{p}\left(\mathrm{x}, \Omega, \Omega^{\prime}\right) \mathrm{d} \Omega^{\prime}>\mathrm{c}>0, \mathrm{x} \in \Gamma, \Omega \in \mathrm{s}$. 
Physically, this requirement means that absorption dominates production by fission, i.e., that $\sigma_{a}>(u-1) \sigma_{f^{\prime}}$ where $\sigma_{a}$ and $\sigma_{f}$ are the absorption and fission cross sections, and $v$ is the mean number of neutrons produced produced per fission. This inequality is always satisfied in a non-multiplying medium $\left(r_{f}=0\right)$ as lcng as some absorption is present $\left(\sigma_{a}>0\right)$; however, it may not be satisfied in some nuclear reactor applications.

Suppose that for each $g \in C(X)$ the equation $T \phi=g$ has a unique solution $\phi=T^{-1} g$ such that $\phi \geq 0$ if $g \geq 0$. Then $\mathrm{T}^{-1}$ is a positive operator from $C(x)$ to an appropriate solution space. Let $e(x, \Omega) \equiv 1$ on $x$. Then (6.2) is equivalent to $\mathrm{Te}>c>0$ on $\mathrm{x}$.

By an elementary argument, $\phi=\mathrm{T}^{-1} \mathrm{~g}$ is bounded for each $g \in c(X)$ and $\|\phi\|<c^{-1}\|g\|$ in terms of the sup norm. Thus, $T^{-1}$ maps $C(X)$ onto a subspace of the bounded functions on $x, T^{-1}$ is bounded, and $\left\|T^{-1}\right\|=\left\|T^{-1} \in\right\|<1 / c$.

The discrete ordinates approximations $\phi_{n}$ satisfy

$$
T_{n} \phi_{n}=g, \quad T_{n}=D-L_{n} .
$$

As above, suppose that $\mathrm{T}_{\mathrm{n}}^{-1}$ exists and is a positive operator on $C(X)$. Since $L_{n} \rightarrow L$, we have $T_{n} \rightarrow T$ and, in particular, $T_{n} e \rightarrow T e$. Therefore, for $n$ sufficiently large, 
(6.5)

$$
T_{n} e>c: 0 \text { on } x,
$$

$T_{n}^{-1}$ is bounded and $\left\|T_{n}^{-1}\right\|<1 / c$. Now,

$$
T_{n}^{-1}-T^{-1}=T_{n}^{-1}\left(T-T_{n}\right) T^{-1} \text {. }
$$

Let $\phi=T^{-1} g$ and $\phi_{n}=T_{n}^{-1} g$. Then

$$
\begin{aligned}
& \phi_{n}-\phi=T_{n}^{-1}\left(T-T_{n}\right) \phi, \\
& \left\|\phi_{n}-\phi\right\| \leq\left\|T_{n}^{-1}\right\|\left\|T_{n} \phi-T \phi\right\| . \\
& \left\|\phi_{n}-\phi\right\|-0 .
\end{aligned}
$$

The error bound for $\left\|\phi_{\mathrm{n}}-\phi\right\|$ is of mixed type, neither purely theoretical nor purely practical.

A special case of this scheme was carried out by Madsen [14] in a classical analysis setting.

7. A rime-Dependent Transport Problem

Wilson [20] investigated the discrete ordinates method for a generalized transport equation in several space dimensions:

$$
\begin{gathered}
\text { (7.1) } \frac{\partial \phi(t, x, v)}{\partial t}+v \cdot \nabla \phi(t, x, v)+\|v\|_{2} \sigma(t, x, v) \phi(t, x, v) \\
=\int_{B} p\left(t, x, v, v^{\prime}\right) \phi\left(t, x, v^{\prime}\right) d v^{\prime}+g(t, x, v) .
\end{gathered}
$$

Independent variables are time $t \in(0, T)$, position $x \in \Gamma$, a rectangle in $R^{n}$, and velocity $v \in R$, a ball in $R^{n}$. The gradient acts with respect to $x$. An 
initial condition specifies $\phi(0, x, v)$. Partial specular reflection on $\partial \Gamma$, ranging from full reflection to an exterior vacuum, was assumed by wilson.

In operator form (7.1) becomes $D_{\phi}=\mathrm{L} \phi+\mathrm{g}$. Discrete ordinate: approximations $\phi_{n}$ satisfy equations $D \phi_{n}=L_{n} \phi_{n}+g$, where $L_{n}$ is a numerical integration approximation to $I$ with a convergent positive quadrature rule.

Wilson [19] obtained existence and uniqueness tr.eorems for weak and strong solutions of a general problem which include $=$ both $D \phi=L \phi+g$ and $D \phi_{n}=L_{n} \phi_{n}+g$ as special cases. The analysis involves an equivalent formulation of the problem as an integral equation which is of volterra type in $t$. Direct iteration yields the existence and uniqueness of $\phi$ and $\phi_{n}$, plus inequalities of the form

$$
\left\|\phi_{n}(t)\right\| \leq\left(a\left\|\phi_{n}(0)\right\|+b\|g\|\right) e^{\left(c\|\sigma\|+d\left\|L_{n}\right\|\right) t},
$$

with $\max$ norms on the functions over undisplayed arguments.

$$
\text { Since } D\left(\phi_{n}-\phi\right)=L_{n}\left(\phi_{n}-\phi\right)+L_{n} \phi-L \phi \text { with zero }
$$

initial conditions and the same boundary conditions, (7.2) implles

$$
\begin{aligned}
& \text { (7.3) }\left\|\phi_{n}(t)-\varphi(t)\right\| \leq b\left\|L_{n} \phi-L\right\| e^{\left(c\|\sigma\|+d\left\|L_{n}\right\|\right) t}, \\
& \text { (7.4) } \phi_{n} \rightarrow \phi \quad \text { uniformly as } n \rightarrow \infty .
\end{aligned}
$$

The error bound in (7.3) is of mixed type. 


\section{Conclusions}

In the precoding, we have attempted to survey developments in the investigation of convergence properties and error bounds for the discrete ordinates approximations to the transport equation. This summairy has been carried out in an abstract setting which, we believe, serves to unify and clarify much previous work in this area. The direction of future investigations is aiso clearly indicated.

While the first studies of convergence properties of the discrete ordinates methods were carried out nearly fifteen years ago, in an astrophysical setting, they have generally not come to the attention of workers interested in practical applications of the method to nuciear ceactor computational problems. In fact, as recently as 1958, a standard work on reactor computing methods refers to the convergence of the discrete ordinates method as an unsolved problem.

A number of practical implications follow from the results surveyed above. For example, the equivalence of the spherical harmonics $\left(\mathrm{P}_{\mathrm{N}}\right)$ and Gauss-quadrature methods for slab geometry transport problems (in the sanse that the solutions agree at the quadrature points) establishes the convergence of, and provides error bounds for, the $P_{N}$ solutions at the quadrature points.

Hopefully, future extensions of the results surveyed here to include three-dimensional systems and discretization of the spatial variabls will provide convergence proofs and practical error estimates which will be of use even in the most complicated practical applications of discrete ordinates methods. 


\section{References}

1. Anselone, P. M. Integral Equatiors of the ScinarzschildMilne Type. J. Math. and Meck., ? (1958), pp. 557-570.

2. Anselone, P. M. Convergence of the Wick-Chandrasekhar Approximation Technique in Radiative Transfer. Astrophys. J., $128(1958)$, pp. 124-i29.

3. Anselone, P. M. Convergence of the Wick-Chandrasekhar Approximation Technique in Radiative Transfer, II. Astrophys. J ., 130 (1959), pp. 881-883.

4. Anselone, P. M. Convergence oj: Chandrasekhar's Method for Inhomogeneous Transfer Problems. J. Math. and Mech., 10 (1961), pp. 537-546.

5. Anselone, P. M. Convergence of Chandrasekhar's Method for the Problem of Diffuse Reflection. Royal Astr. Soc. Monthly Notices, 120 (1960), pp. 498-503.

6. Anselone, P. M. Collectively Compact Operator Aprroximation Theory and Applications to Integral Equations. Prentice-Hall, Inc., Englewood Cliffs, N. J., 1971.

7. Chandrasekhar, S. On the Radiative Equilibrium of a Stellar Atmosphere, II. Astrophys. J., 100 (1944), pp. 76-86.

8. Chandrasekhar, S. Radiative Transfer. Oxford University Press, 1950 .

9. Hopf, E. Mathematical Problems of Radiative Equilibrium. Cambridge University Press, 1934. 
10. Keller, H. B. Approximate Solutions of Transport Problems. SIAM J. Appl. Math., 8 (1960), pp. 43-73.

11. Keller, H. B. On the Pointw:-se Convergence of the Discrete Ordinate Method. SIAM J. Appl. Math., 8 (1960), pp. 560-567.

12. Keller, H. B. Convergence of the Discrete Ordinate Method for the Anisotropic Scattering Transport Equation. Provisional Intl. Comp. Center, Rome Symposium, Birkhauser-Verlag, Basel/Stuttgart, 1960, pp. 292-299.

13. Kofink, W. Studies of the Spherical Harmonics Method in Neutron Transport Theory, Nuovo Cimento 10, 9 (1958), Supplemento, pp. 497-541.

14. Madsen, N. K. Pointwise Convergence of the ThreeDimensional. Discrete Ordinate Method. SIAM J. Num. Anal., 8 (1971), pp. 266-269.

15. Nelson, P. Convergence of the Discrete Ordinates Method for inisotropically Scattering wultiplying Particles in a Subcritical Slab. SIAM J. Nur. Anal., 10 (1973), pp. 175-181.

16. Nestell, M. K. The Convergence of the Discrete Ordinates Method for integral Equations of Anisotropic Radiative Transfer. Tech. Report 23, Math Dept., Oregon State University, 1965.

17. Wendroff, B. On the Convergence of the Discrete Ordinate Method. SIAM J. Appl. Math., 8 (1960), FP. 508-511. 
18. Wick, G. C. Uber Ebene Diffusions Problem. Z. für Physik 121 (1943), pp. 702-718.

19. Wilson, D. G. Time-Dependent Linear Transport, I., J. Math. Anal. Appl., to appear.

20. Wilson, D. G. Time Dependent Linear Transport, III; Convergence of Discrete Ordinate Method, submitted for publivation. 